\title{
Low tumor glutathione level as a sensitivity marker for glutamate-cysteine ligase inhibitors
}

\author{
SATORU NISHIZAWA ${ }^{1}$, HIDEO ARAKI ${ }^{1}$, YOSHINORI ISHIKAWA ${ }^{1}$, SATOSHI KITAZAWA ${ }^{1}$, \\ AKITO HATA $^{2}$, TOMOYOSHI SOGA ${ }^{3}$ and TAKAHITO HARA ${ }^{1}$ \\ ${ }^{1}$ Oncology Drug Discovery Unit and ${ }^{2}$ Biomolecular Research Laboratories, \\ Pharmaceutical Research Division, Takeda Pharmaceutical Company Limited, Fujisawa, \\ Kanagawa 251-8555; ${ }^{3}$ Institute for Advanced Bioscience, Keio University, Tsuruoka, Yamagata 997-0052, Japan
}

Received January 31, 2017; Accepted April 13, 2017

DOI: $10.3892 / \mathrm{ol} .2018 .8447$

\begin{abstract}
Previous metabolomic analyses of cancer have revealed elevated glutathione levels in tumors. An inhibitor of cystine uptake was identified to suppress glutathione biosynthesis, leading to ferroptosis, a novel iron-dependent form of cell death that differs from apoptosis and necrosis. Glutamate-cysteine ligase (GCL) is the rate-limiting enzyme in the glutathione biosynthesis pathway. Buthionine sulfoximine (BSO), a GCL inhibitor, has previously demonstrated limited clinical benefits. Therefore, selecting patients who respond well to the inhibitor is a key approach for successful future drug development. Ferroptosis induction by BSO has not been fully examined in prior studies. Therefore, the present study investigated the pharmacological effects of BSO and the association between basal intracellular glutathione levels and sensitivity to BSO in cultured cell lines derived from various types of cancer, including those of the kidney [769P, 786-O, A-498, A704, ACHN, Caki-1, Caki-2, G401, G402, RCC4 VHL(-/-), RCC4 VHL(+/+), SK-NEP-1 and SW156] and ovaries (A2780 and A2780/CDDP). BSO was demonstrated to suppress glutathione levels and induce lipid peroxidation, thereby inhibiting cell viability. The viability-reducing effects of BSO were attenuated by ferroptosis inhibition and enhanced by iron, indicating that BSO induced ferroptosis in cancer cells. The cell lines sensitive to BSO, including G402, tended to exhibit non-significantly lower levels of glutathione compared with the BSO-insensitive cell lines, including Caki-2 $(\mathrm{P}=0.08)$. Patient sample data indicated the existence of a population of colorectal tumors with lower glutathione levels compared with those of matched normal tissues that might be suitable for the clinical testing of sensitivity to GCLC inhibitors. Collectively,
\end{abstract}

Correspondence to: Dr Takahito Hara, Oncology Drug Discovery Unit, Pharmaceutical Research Division, Takeda Pharmaceutical Company Limited, 26-1 Muraokahigashi 2-chome, Fujisawa, Kanagawa 251-8555, Japan

E-mail: takahito.hara@takeda.com

Key words: cancer, buthionine sulfoximine, ferroptosis, glutathione, iron these data suggest that GCL inhibition leads to ferroptosis in cancer cells, and that low glutathione tumor levels may be a patient selection marker for the use of GCL inhibitors in the treatment of tumors.

\section{Introduction}

Metabolic alterations in cancer cells can enhance cell growth and survival by promoting energy metabolism (1-3). In addition, previous metabolomic analyses of colorectal and kidney cancer cells have revealed increased levels of reduced glutathione $(\mathrm{GSH})$ in tumors along with changes in glycolysis, amino acid metabolism and the tricarboxylic acid cycle (4-7). These observations suggest that the GSH-dependent defense system against reactive oxygen species (ROS) serves a critical role in these types of cancer. ROS were recently demonstrated to induce ferroptosis, which is an iron-dependent form of non-apoptotic and non-necrotic cell death (8-10). Ferrostatin-1 has been identified as a compound that attenuates ferroptosis by blocking lipid peroxidation $(11,12)$. Erastin, a cystine uptake inhibitor, is hypothesized to induce ferroptosis by suppressing the synthesis of GSH, leading to lipid oxidation (8).

Glutamate-cysteine ligase (GCL; EC 6.3.2.2), composed of a GCL catalytic subunit (GCLC) and GCL modifier subunit (GCLM), is the rate-limiting enzyme in GSH biosynthesis, and is responsible for converting glutamine and cysteine to $\gamma$-glutamylcysteine (13). Buthionine sulfoximine (BSO) is a GCLC inhibitor. Ferroptosis induction by BSO in cancer cells has not been fully clarified. In an early clinical trial, BSO was identified to deplete tumor glutathione levels when administered by continuous infusion but did not demonstrate clinical benefits against cancer (14). However, targeting sensitive cancer cell types that have been identified using markers for GCLC inhibitor-sensitivity may optimize the effects of these drugs. Therefore, the present study investigated whether BSO induced ferroptosis in cancer cells, and whether the cellular glutathione level may be a marker for GCLC inhibitor-sensitivity.

\section{Materials and methods}

Cell-free GCLC enzymatic assay. Expression of N-terminal His-tagged human GCLM was induced with $1 \mathrm{mM}$ 
isopropyl- $\beta$-D-thiogalactoside (IPTG, Wako Pure Chemical Industries, Ltd., Osaka, Japan) at $30^{\circ} \mathrm{C}$ for $5 \mathrm{~h}$ in Escherichia coli. Expression of C-terminal His-tagged human GCLC was induced with $1 \mathrm{mM}$ IPTG at $16^{\circ} \mathrm{C}$ for $16 \mathrm{~h}$ in Escherichia coli. GCLC and GCLM proteins were purified by Ni-NTA affinity chromatography (Qiagen, Hilden, Germany), followed by Superdex 200 gel filtration chromatography (GE Healthcare, Piscataway, NJ, USA), as previously described (15). Following purification, the enzymes were used for the subsequent studies. 0.1, 1, 10, and $100 \mu \mathrm{M}$ of BSO (Sigma-Aldrich; Merck KGaA, Darmstadt, Germany) was premixed with enzymes (10 $\mathrm{nM}$ for each) for $30 \mathrm{~min}$ prior to the addition of $200 \mu \mathrm{M}$ adenosine triphosphate (ATP), $1.2 \mathrm{mM}$ glutamic acid and $200 \mu \mathrm{M}$ cysteine. Following incubation for $60 \mathrm{~min}$, the reaction was terminated by adding $1 \%$ formic acid solution and the ATP and $\gamma$-glutamylcysteine levels were measured using RapidFire 300 mass spectrometry (Agilent Technologies, Inc., Santa Clara, CA, USA) coupled with API4000 triple quadrupole mass spectrometer (AB Sciex, Framingham, MA). The analytical data were integrated using RapidFire Integrator software (version 4.0; Agilent Technologies, Inc.).

Cell lines. The cell lines used in the present study were purchased from American Type Culture Collection (Manassas, VA, USA), DS Pharma Biomedical (Osaka, Japan), and Horizon Discovery Ltd. (Cambridge, UK). The cells were maintained at $37^{\circ} \mathrm{C}$ in an atmosphere of $5 \% \mathrm{CO}_{2}$ in RPMI-1640 (Thermo Fisher Scientific, Inc., Waltham, MA, USA) with $10 \%$ fetal bovine serum (FBS; Thermo Fisher Scientific, Inc.). All cell lines used are summarized in Table I.

Viability assays and determination of cellular glutathione. The cells were seeded at 1,000-3,000 cells/100 $\mu \mathrm{l}$ in each well of a 96-well plate. The following day, BSO, GSH monoethyl ester (GSHee; Bachem AG, Bubendorf, Switzerland), ferrostatin-1, N-acetylcysteine (NAC; both from Sigma-Aldrich; Merck KGaA), cisplatin and ferric ammonium citrate (FAC; both from Wako Pure Chemical Industries, Ltd.) were added to the wells. After a 24-h incubation, the cellular total glutathione level [including GSH and glutathione disulphide (GSSG)] was determined using a GSH/GSSG-Glo Assay (Promega Corporation, Madison, WI, USA). Following a 3-day incubation, cell viability was assessed using a Cell Titer-Glo Luminescent Cell Viability Assay (Promega Corporation). To analyze the basal levels of total glutathione (GSH+GSSG) without BSO treatment, the total glutathione levels and cell viability were measured 2 days after the cells were plated.

Analysis of mutations and copy number of the von Hippel-Lindau tumor suppressor (VHL) gene in cancer cell lines. GSH is oxidized into GSSG when neutralizing ROS (16). GSSG may be reduced into GSH by glutathione reductase using NADPH (17) whose major source is pentose phosphate pathway (PPP) (18). PPP branches from glycolysis (18) that is known to be regulated by various cancer associated genes including hypoxia-inducible factor $1-\alpha$ (19-21) whose expression is often upregulated by $V H L$ deficiency (22). Therefore, VHL status is potentially associated with the regulation of the ROS defense system by GSH. In order to examine the association between VHL status, BSO sensitivity and glutathione levels, the VHL status of cancer cells were analyzed. $V H L$ mutation data were downloaded from the Catalog of Somatic Mutations in Cancer database, Cell Lines Project v79 (ftp://ftp. sanger.ac.uk/pub/CGP/cosmic). The copy number data for $V H L$ were downloaded from the Cancer Cell Line Encyclopedia (http://www.broadinstitute.org/ccle).

Measurement of lipid peroxidation. A total of $1 \times 10^{6}$ PANC-1 cells were seeded in a $10-\mathrm{cm}$ dish, treated with BSO the following day, and incubated for $24 \mathrm{~h}$ at $37^{\circ} \mathrm{C}$. Subsequently, the cells were stripped with $0.25 \%$ trypsin at $37^{\circ} \mathrm{C}$. The cells were incubated with $5 \mu \mathrm{M}$ BODIPY 581/591 C11 Lipid Peroxidation Sensor (Thermo Fisher Scientific, Inc.) for $30 \mathrm{~min}$. Following two washes with PBS, the cells were re-suspended in BD FACS flow sheath fluid (BD Biosciences, San Jose, CA, USA). The lipid peroxidation level was assessed using FACS Verse ${ }^{\mathrm{TM}}$ system and analyzed with FAC Suite v1.0.5.3841 (both BD Biosciences).

Metabolomic analysis of colorectal tumors and cell lines. As described in the previous report (23), all the experiments were conducted according to a study protocol approved by the Institutional Ethics Committee of Kagawa University (Heisei 24-040) upon obtaining informed consent from all subjects. The tumor and normal tissues were surgically obtained from 275 colorectal cancer patients who had not received any prior treatments in Kagawa University Hospital from January 2012 to December 2013 according to the methods of the previous report (23). Of the 275 patients, $5(1.8 \%), 2$ (0.7\%), 36 (13.1\%), 102 (37.1\%), 85 (30.9\%), 45 (16.4\%), had adenoma (median age, 77 years; range, 52-84 years; male/female, 1:4) and a clinical stage of 0 (median age, 73 years; range, 73-74 years; male/female, 1:1), I (median age, 70 years; range, 35-89 years; male/female, 22:14), II (median age, 73 years; range, 35-96 years; male/female, 64:38), III (median age, 70 years; range, 28-92 years; male/female, 42:43), IV (median age, 67 years; range, 37-88 years; male/female, 25:20), respectively. The absolute amounts of metabolites in clinical colorectal tumor samples $(n=275)$, their matched normal tissues $(\mathrm{n}=275)$ (23) and cell lines (RCC4 $\mathrm{VHL}^{-/-}$and RCC4 $\mathrm{VHL}^{+/+}$) were measured using capillary electrophoresis-triple quadrupole/time-of-flight MS at Keio University (Tsuruoka, Japan), according to the methods of Yuan et al (24) and Soga et al (25-27).

SDS-PAGE and western blot analysis. The anti-heat-shock protein 90 antibody (cat no. CST4877; dilution, 1:2,000) for western blotting was purchased from Cell Signaling Technology, Inc. (Danvers, MA, USA). Antibodies against GCLC (cat no. ab190685; dilution, 1:5,000) and GSH synthetase (GSS; cat no. ab124811; dilution, 1:2,000) were purchased from Abcam (Cambridge, MA, USA). Cells (DLD-1, HCT-116, MIA PaCa-2, PC-3, 769P, 786-O, A-498, A704, ACHN, Caki-1, Caki-2, G401, G402, RCC4 $\mathrm{VHL}^{-/ \text {, }}$ RCC4 $\mathrm{VHL}^{+/+}$, SK-NEP-1, SW156) were lysed in SDS sample buffer (Bio-Rad Laboratories, Inc., Hercules, CA, USA) and heated at $95^{\circ} \mathrm{C}$ for $5 \mathrm{~min}$. Cell lysates $(3 \mu \mathrm{g})$ were separated using SDS-PAGE (7.5-15\% gradient gel) and transferred onto Sequi-Blot ${ }^{\mathrm{TM}}$ polyvinylidene fluoride membranes (Bio-Rad Laboratories, Inc.). The membranes were blocked 
Table I. All cancer cell lines used in the present study.

\begin{tabular}{|c|c|c|c|c|}
\hline Cell line & Organ & Supplier & Catalog number & Experimental use \\
\hline $769 \mathrm{P}$ & Kidney & ATCC & CRL-1933 & Fig. 3 \\
\hline $786-\mathrm{O}$ & Kidney & ATCC & CRL-1932 & Table II; Fig. 3 \\
\hline A-498 & Kidney & ATCC & HTB-44 & Tables II and III; Fig. 3 \\
\hline A2780 & Ovary & DS pharma (ECACC) & 93112519 & Table II \\
\hline A2780/CDDP & Ovary & DS pharma (ECACC) & 93112517 & Table II \\
\hline A704 & Kidney & ATCC & HTB-45 & Fig. 3 \\
\hline $\mathrm{ACHN}$ & Kidney & ATCC & CRL-1611 & Table II; Fig. 3 \\
\hline Caki-1 & Kidney & ATCC & HTB-46 & Fig. 3 \\
\hline Caki-2 & Kidney & ATCC & HTB-47 & Table II; Fig. 3 \\
\hline COLO 205 & Colon & ATCC & CRL-222 & Table II \\
\hline DLD-1 & Colon & Horizon discovery & HD PAR-086 & Fig. 3 \\
\hline DU 145 & Prostate & ATCC & НTB-81 & Table II \\
\hline G401 & Kidney & ATCC & CRL-1441 & Fig. 3 \\
\hline G402 & Kidney & ATCC & CRL-1440 & Tables II and III; Fig. 3 \\
\hline HCT-116 & Colon & ATCC & CCL-247 & Tables II and III; Fig. 3 \\
\hline HCT-15 & Colon & ATCC & CCL-225 & Table II \\
\hline HT-29 & Colon & ATCC & HTB-38 & Table II; Fig. 2 \\
\hline LS $174 \mathrm{~T}$ & Colon & ATCC & CL-188 & Table II \\
\hline MIA PaCa-2 & Pancreas & ATCC & CRL-1420 & Fig. 3 \\
\hline PANC-1 & Pancreas & DS pharma (ECACC) & 87092802 & Table II; Figs. 1 and 2 \\
\hline $\mathrm{PC}-3$ & Prostate & ATCC & CRL-1435 & Table II; Fig. 3 \\
\hline RCC4 VHL-/- & Kidney & DS pharma (ECACC) & 3112702 & Table II; Fig. 3 \\
\hline RCC4 VHL+/+ & Kidney & DS pharma (ECACC) & 3112703 & Table II; Fig. 3 \\
\hline $\mathrm{RKO}$ & Colon & ATCC & CRL-2577 & Table II \\
\hline SK-NEP-1 & Kidney & ATCC & НTB-48 & Fig. 3 \\
\hline SW156 & Kidney & ATCC & CRL-2175 & Fig. 3 \\
\hline SW48 & Colon & Horizon discovery & HD PAR-006 & Table II; Fig. 2 \\
\hline SW480 & Colon & ATCC & CCL-228 & Table II \\
\hline SW620 & Colon & ATCC & CCL-227 & Table II \\
\hline
\end{tabular}

ATCC, American Type Culture Collection; ECACC, European Collection of Authenticated Cell Cultures.

with Starting Block ${ }^{\mathrm{TM}}$ T20 PBS Blocking Buffer (Thermo Fisher Scientific, Inc.) and probed overnight at $4^{\circ} \mathrm{C}$ with the primary antibodies diluted with $10 \%$ Blocking Ace (DS Pharma Biomedical) in PBS containing 0.1\% Tween-20. The membranes were subsequently washed with PBS containing $0.1 \%$ Tween-20 (Wako Pure Chemical Industries, Ltd.) and incubated for one hour at room temperature with horseradish peroxidase-labeled secondary antibody (Cell Signaling Technology; cat. no. 7074; dilution 1:3,000) diluted with Can Get Signal ${ }^{\circledR}$ immunoreaction enhancer solution II. The membrane was washed with PBS containing $0.1 \%$ Tween-20 three times for $10 \mathrm{~min}$, and chemiluminescence was used to detect the antibody-labeled proteins using SuperSignal West Femto Maximum Sensitivity Substrate (Thermo Fisher Scientific, Inc.) and detected with the LAS-3000 Luminescent Image Analyzer (Fujifilm Holdings Corporation, Tokyo, Japan).

Gene expression analysis of tumors from patients with colorectal cancer. The levels of gene expression of GCLC and
GSS in colorectal tumors $(n=41)$ and their matched normal tissues $(n=39)$ were analyzed using the Agilent Expression Array Sure Print G3 Human Gene Expression v2 8x60K Microarray (Agilent Technologies, Inc.) at Keio University (16).

Statistical analysis. The half-maximal inhibitory concentration $\left(\mathrm{IC}_{50}\right)$ values in the GCLC enzymatic assays were determined using the XLfit software 5.4.0.8 (IDBS, Guildford, UK) or GraphPad Prism v5.01 (GraphPad Software, Inc., La Jolla, CA, USA). The $\mathrm{IC}_{50}$ values of the viability studies were determined using a nonlinear regression curve fitted using GraphPad Prism v.6.01. Differences in cell viability and rescue assays between the control and treatment groups were analyzed using a Williams' test, and the combination effects were evaluated using a two-way analysis of variance followed by a Tukey's test. Correlation between glutathione levels and growth inhibition by $100 \mu \mathrm{M}$ of BSO in cancer cells was evaluated using Pearson correlation analysis. Correlations between basal glutathione levels and GCLC or GSS protein levels in cancer cells were determined by linear regression 
A

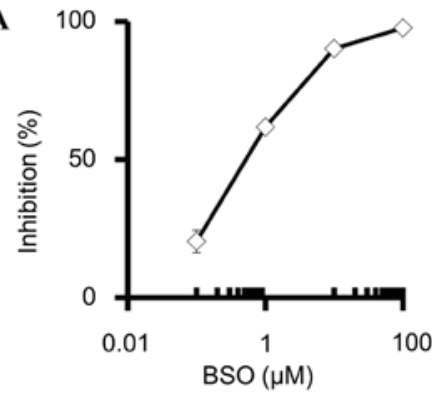

C
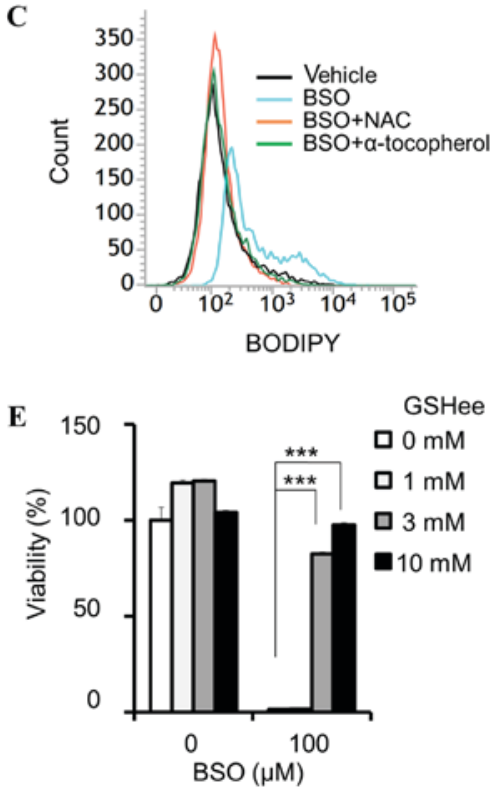

B

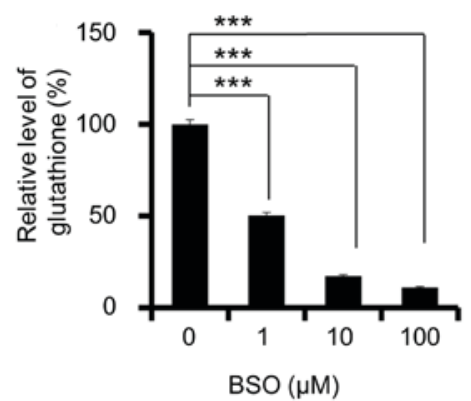

D

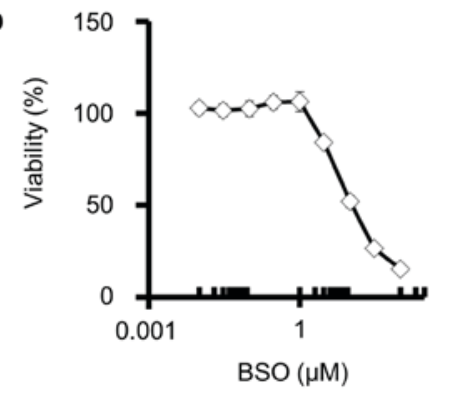

$\mathbf{F}$

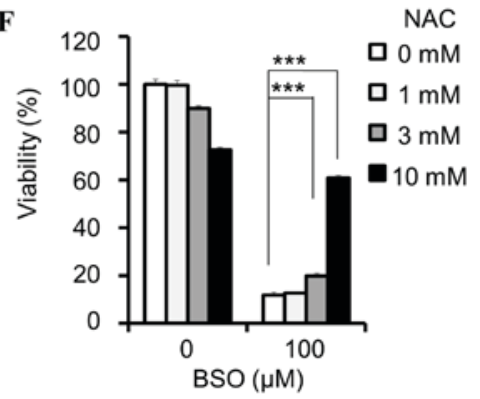

Figure 1. BSO suppresses glutathione biosynthesis and decreases cell viability. (A) Enzymatic inhibition of GCL by BSO. (B-F) Cellular effects of BSO in PANC-1 cells. (B) GSH+GSSG levels, normalized by cellular ATP, determined following incubation with BSO for $24 \mathrm{~h}$. (C) Induction of lipid peroxidation by $100 \mu \mathrm{M}$ BSO, and attenuation by $10 \mathrm{mM} \mathrm{NAC}$ and $100 \mu \mathrm{M} \alpha$-tocopherol. (D) BSO-induced decrease in cell viability, and rescue effects of (E) GSHee and (F) NAC. (A, n=4 and D, n=3) Data are presented as the mean \pm SD. (B, E and F) Data are presented as the mean \pm SD $(n=3)$; ${ }^{* * *} P<0.0005$ using Williams' test. BSO, buthionine sulfoximine; GCL, glutamate-cysteine ligase; GSH, glutathione (reduced form); GSSG, glutathione disulphide; ATP, adenosine triphosphate; NAC, N-acetylcysteine; GSHee, GSH monoethyl ester; SD, standard deviation; BODIPY, boron dipyrromethene.

analysis. Correlation between $\log _{2}(\mathrm{~T} / \mathrm{N})$ values of GSH and total glutathione (GSH+GSSG) in tissue samples from patients with colorectal cancer was evaluated by Pearson correlation analysis. Correlations between total glutathione (GSH+GSSG) and GCLC or GSS mRNA levels (T/N) in tissue samples from patients with colorectal cancer were also evaluated by Pearson correlation analysis. $\mathrm{P}<0.025$ was considered to indicate a statistically significant difference in the statistical tests for rescue studies. In the rest of the statistical tests, $\mathrm{P}<0.05$ was considered to indicate a statistically significant difference in all statistical tests other than the rescue studies.

\section{Results}

Pharmacological properties of BSO. In the cell-free GCLC enzymatic assay, BSO inhibited the activity of GCLC with an $\mathrm{IC}_{50}$ of $570 \mathrm{nM}$ [95\% confidence interval (CI) 429-757 nM; Fig. 1A]. BSO reduced the total glutathione (GSH+GSSG) levels in PANC-1 cells (Fig. 1B) and induced lipid peroxidation, which was attenuated by NAC and $\alpha$-tocopherol (Fig. 1C). In addition, BSO decreased the viability of PANC-1 cells (Fig. 1D), and this effect was attenuated by the addition of a membrane-permeable GSH derivative, GSHee (Fig. 1E), and NAC (Fig. 1F). These results indicate that cell viability was inhibited by the suppression of intracellular glutathione and the subsequent lipid peroxidation.

BSO induces ferroptosis. GSH reduction has been identified to induce ferroptosis, which can be reversed by ferrostatin-1 $(11,12)$. In the present study, the viability-reducing effect of BSO on PANC-1 cells was rescued by ferrostatin-1 (Fig. 2A), indicating that BSO induces ferroptosis in cancer cells. In addition, ferroptosis is hypothesized to depend on intracellular iron concentration (8); therefore, the present study examined the effects of iron on BSO-induced inhibition. FAC synergistically enhanced the BSO-induced inhibition of PANC-1 (Fig. 2B) and HT-29 (Fig. 2C) cell viability. These results indicate that the inhibitory effects of BSO are iron-dependent. The BSO-induced inhibition of SW48 cell viability was attenuated by NAC (Fig. 2D), but not ferrostatin-1 (Fig. 2E).

Sensitivity of cancer cell lines to BSO. Cell panel viability assays were conducted, and various colorectal, kidney, 
A

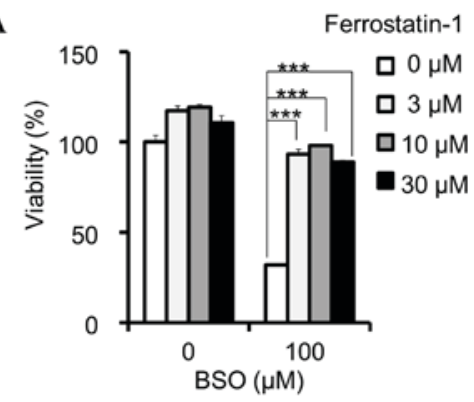

C
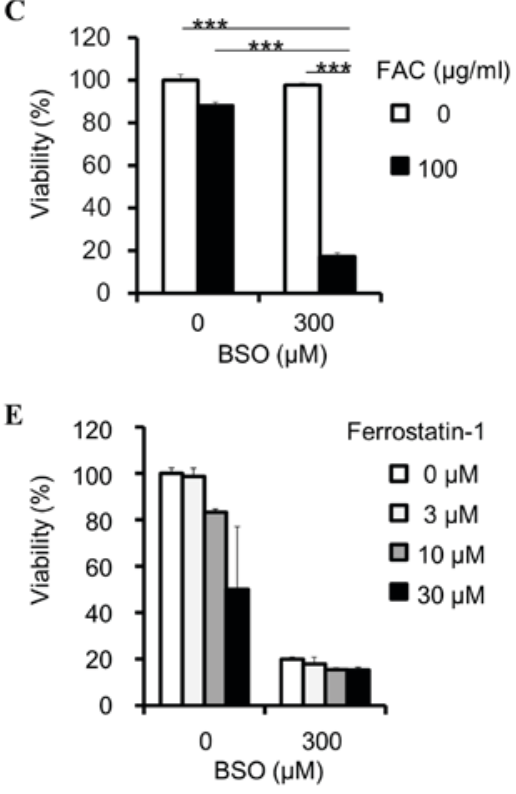

B

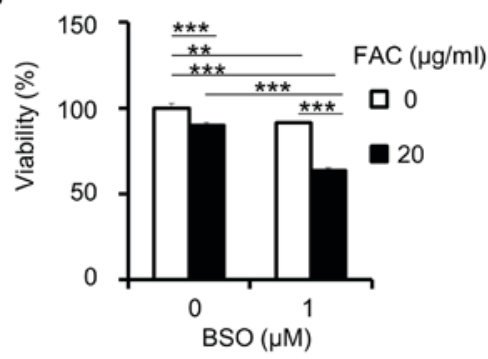

D

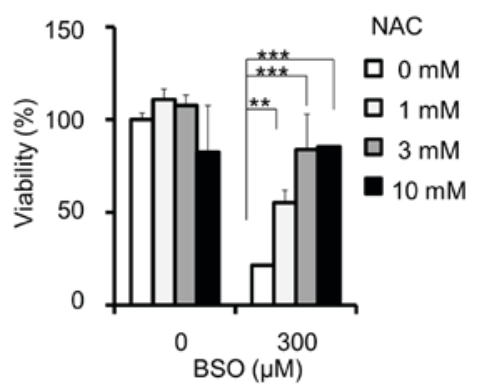

Figure 2. BSO induces ferroptosis in cancer cells. (A) Rescue effects of ferrostatin-1 against BSO-induced decrease of cell viability in PANC-1 cell lines; ${ }^{* * * *} \mathrm{P}<0.0005$ using Williams' test. (B) Effects of FAC on viability reduction by BSO in PANC-1 cells, compared by two-way ANOVA: BSO and FAC interaction, $\mathrm{P}<0.001, \mathrm{~F}=69.56$; $\mathrm{BSO}, \mathrm{P}<0.001, \mathrm{~F}=266.89$; FAC, $\mathrm{P}<0.001, \mathrm{~F}=312.01$; Tukey's post hoc test, ${ }^{* * *} \mathrm{P}<0.01$ and ${ }^{* * * *} \mathrm{P}<0.001$. (C) Effects of FAC on viability reduction by BSO in HT-29, compared by two-way ANOVA: BSO and FAC interaction, $\mathrm{P}<0.001$, F=157.96; BSO, P<0.001, F=179.84; FAC, P<0.001, F=287.57; Tukey's post hoc test, ${ }^{* * *} \mathrm{P}<0.001$. Effects of (D) NAC and (E) ferrostatin-1 on cell viability reduction by BSO in SW48 cells; ${ }^{* *} \mathrm{P}<0.005$ and ${ }^{* * *} \mathrm{P}<0.0005$ using Williams test. Data are presented as the mean \pm standard deviation $(\mathrm{n}=3)$. BSO, buthionine sulfoximine; FAC, ferric ammonium citrate; ANOVA, analysis of variance; NAC, $\mathrm{N}$-acetylcysteine.

pancreatic and ovarian cancer cell lines demonstrated high sensitivity to BSO (Table II). To examine whether the glutathione levels may be a sensitivity marker for BSO, the correlation between the basal levels of total glutathione (GSH+GSSG) and sensitivity to BSO of cancer cells was investigated. BSO-sensitive cells (G402, RCC4 $\mathrm{VHL}^{-/-}$, and A-498) tended to exhibit lower glutathione levels $(\mathrm{P}=0.08)$ compared with those of insensitive cells ( RCC4 $\mathrm{VHL}^{+/+}$, Caki-2, and HCT-116) (Fig. 3A). GCLC inhibition suppresses cellular glutathione levels (Fig. 1B); therefore, the differences in glutathione levels among cancer cells may be attributable to different protein levels of enzymes in the GSH biosynthesis pathway. The correlation between glutathione levels and the protein levels of GCLC or GSS were examined, and it was identified that GCLC protein and glutathione levels were positively correlated $\left(\mathrm{r}^{2}=0.814, \mathrm{P}=0.04\right)$ in cancer cells (Fig. 3B and C). By contrast, GSS protein and glutathione levels were not correlated $\left(\mathrm{r}^{2}=0.021, \mathrm{P}=0.82\right.$; Fig. $3 \mathrm{~B}$ and $\left.\mathrm{C}\right)$.

RCC4 plus vector ( $\mathrm{RCC} 4 \mathrm{VHL}^{-/}$) kidney cancer cells, which do not express $V H L$, were more sensitive to BSO compared with isogenic RCC4 plus VHL [RCC4 VHL(+/+)] cells which do express $V H L\left(\log \mathrm{IC}_{50},-4.77\right.$ vs. $-4.0 \mathrm{M}$, respectively; Table II).
Total glutathione and GSH levels were lower in RCC4 $\mathrm{VHL}^{-1-}$ cells compared with RCC4 $\mathrm{VHL}^{+/+}$cells (Fig. 3A and D). The association between $V H L$ status, BSO sensitivity and glutathione levels was additionally investigated using G402 (VHL wild-type), HCT-116 (VHL wild-type), VHL-deficient A498 (Fig. 3E; Table III) and VHL-mutant Caki-2 cells. However, no clear correlation was observed between the VHL status and sensitivity to BSO, or VHL status and glutathione levels in these cancer cell lines (Table II; Fig. 3A).

Glutathione levels in tumors from patients with colorectal cancer. To examine the occurrence of tumors in patients with low glutathione levels, the total glutathione and GSH levels in tumors and their matched normal tissues from the patients with colorectal cancer were measured. The glutathione level was upregulated in the majority of tumors compared with that in the matched normal tissues; however, 15\% (44/284) of the tumor samples demonstrated lower glutathione levels compared with those of the matched normal tissues (Fig. 4A). Total glutathione and GSH levels were positively correlated $\left(r^{2}=0.669, \mathrm{P}=8.18 \times 10^{-66}\right.$; Fig. 4B). In addition, the correlation between GCLC and GSS mRNA expression and glutathione 
levels was examined in tumors from patients with colorectal cancer, and no marked correlation was observed (Fig. 4C).

\section{Discussion}

In clinical trials, BSO has been shown to deplete glutathione in tumors, but has not demonstrated substantial therapeutic benefits $(13,28)$. Thus, selecting sensitive cancer types and patients using sensitivity markers may enhance the clinical efficacy of anticancer therapies (29-31). However, sensitive cancer types and sensitivity markers for GCLC inhibitors remain incompletely characterized. Therefore, the present study investigated potentially BSO-sensitive cancer cell types and a sensitivity marker for GCLC inhibitors using BSO in cultured cancer cells. In a cell viability assay, colorectal (SW48), kidney (G402, RCC4 $\mathrm{VHL}^{-1-}$ and 786-O), pancreatic (PANC-1), and ovarian (A2780 CDDP) cancer cells demonstrated sensitivity to BSO, suggesting that treatment with a GCLC inhibitor may be beneficial for these cancer types. The potential sensitivity of kidney cancer to GCLC inhibitors is supported by the present study, in which, kidney cancer cell lines were identified to be vulnerable to erastin (8). Subsequently, the association between basal intracellular glutathione levels and cellular sensitivity to BSO was investigated, and it was demonstrated that BSO-insensitive cell lines tended to exhibit lower glutathione levels compared with the sensitive cells. These results suggest that low glutathione tumor levels may be a sensitivity marker for GCLC inhibitors.

Furthermore, the analysis of tumors and their matched normal tissues from patients with colorectal cancer revealed that $15 \%$ of colorectal cancers exhibited lower glutathione tumor levels compared with those of the matched normal tissues. These low-glutathione-content tumor populations may be useful for examining the clinical benefits of GCLC inhibitors. Glutathione and GSH levels were correlated, suggesting that glutathione may be substituted with GSH. Furthermore, proton and carbon-13 nuclear magnetic resonance has been suggested to be able to detect GSH non-invasively (32). These technologies may be applied during the selection of patients for treatment with GCLC inhibitors. The differences in glutathione levels between cancer cell types may be caused by differences in GCLC protein levels. In the present study, protein levels of GCLC and glutathione were correlated among G-402, RCC4 $\mathrm{VHL}^{-/}, \mathrm{A}-498, \mathrm{RCC} 4 \mathrm{VHL}^{+/+}$, Caki-2, and HCT-116 cells. By contrast, patient tumor samples did not demonstrate a significant correlation between glutathione and GCLC mRNA expression, and differences between GCLC protein levels and GCLC mRNA expression levels may explain this result; however, additional studies are required to elucidate this.

In addition, $V H L$ status was examined as a potential regulator of glutathione levels. Although RCC4 $\mathrm{VHL}^{-1-}$ cells demonstrated lower glutathione levels compared with those of RCC4 $\mathrm{VHL}\left({ }^{+++}\right)$cells, this observation was consistent with the analyses of other cancer cell lines. Therefore, $V H L$ is not likely to be correlated with glutathione levels. Other gene alterations may have obscured the effects of $V H L$ status and, therefore, additional studies are required to clarify the association between $V H L$ status and sensitivity to GCLC inhibitors.

Ferroptosis is a newly-identified type of ROS-induced cell death (8-10), which the cysteine-uptake inhibitor erastin
Table II. Sensitivity of cancer cell lines to buthionine sulfoximine.

\begin{tabular}{llc}
\hline Cell line & Organ & LogIC $_{50}(\mathrm{M})$ \\
\hline G402 & Kidney & -5.73 \\
PANC-1 & Pancreas & -5.33 \\
RCC4 VHL $^{-/}$ & Kidney & -4.77 \\
786-O & Kidney & -4.10 \\
A-498 & Kidney & -4.09 \\
A2780 CDDP & Ovary & -4.05 \\
SW48 & Colon & -4.04 \\
A2780 & Ovary & -3.81 \\
PC-3 & Prostate & $>-3.5$ \\
HCT-15 & Colon & $>-3.5$ \\
SW620 & Colon & $>-3.5$ \\
RCC4 VHL & Kidney & $>-4.0$ \\
COLO 205 & Colon & $>-3.5$ \\
LS 174T & Colon & $>-3.5$ \\
HCT-116 & Colon & $>-3.5$ \\
RKO & Colon & $>-3.5$ \\
HT-29 & Colon & $>-3.5$ \\
SW480 & Colon & $>-3.5$ \\
ACHN & Kidney & $>-3.5$ \\
Caki-2 & Kidney & $>-4.0$ \\
DU 145 & Prostate & $>-3.5$ \\
\hline
\end{tabular}

$\mathrm{IC}_{50}$, half-maximal inhibitory concentration.

Table III. Mutational analysis of von Hippel-Lindau tumor suppressor gene in cancer cell lines using Catalog of Somatic Mutations in Cancer database.

\begin{tabular}{lcc}
\hline Cell line & AA mutation & CDS mutation \\
\hline A-498 & p.G144Fsa14 & c.426-429 del \\
G402 & - & - \\
HCT-116 & - & - \\
\hline
\end{tabular}

AA, amino acid; CDS, coding DNA sequence.

has been shown to induce (8-10). However, whether the inhibition of enzymes involved in GSH biosynthesis induces ferroptosis has not been fully determined. This was addressed in the present study by inhibiting GCLC using BSO, which subsequently induced the lipid peroxidation that is required for ferroptosis (8). In addition, the BSO-induced decrease in cell viability was attenuated by a ferroptosis inhibitor, ferrostatin-1. Furthermore, ferroptosis is dependent on cellular iron (8), and treatment with iron enhanced the BSO-induced reduction in viability of PANC-1 cells, indicating its dependence on iron. These results demonstrated that the inhibition of GCLC by BSO induced ferroptosis PANC-1 cells. Based on its iron-dependence, cancer with high iron levels may be sensitive to GCLC inhibitors, and a combination therapy with iron 

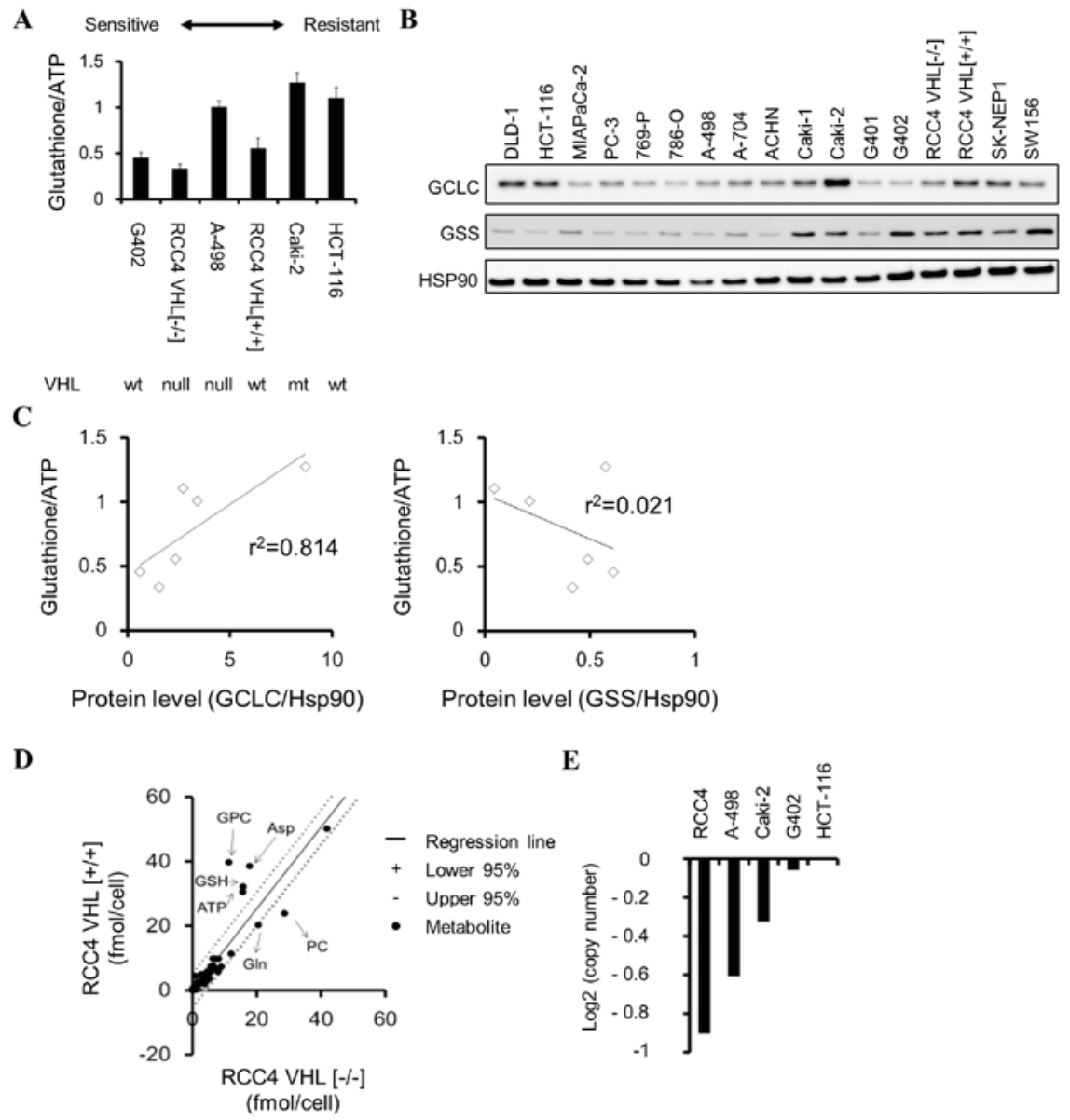

Figure 3. Glutathione levels in cancer cells. (A) Total glutathione (GSH + glutathione disulphide) levels, normalized to cellular ATP levels, in various cancer cell lines. Data are presented as the mean \pm standard deviation $(n=3)$. (B) Western blot analysis of GCLC, GSS and Hsp90 across various cancer cell lines. (C) Correlation between glutathione and GCLC or GSS protein levels in HCT-116, A-498, Caki-2, G402, RCC4 VHL $L^{-1}$, RCC4 VHL ${ }^{+/+}$. Correlations were determined by linear regression analysis ( $\mathrm{P}=0.04$ for glutathione and GCLC; $\mathrm{P}=0.82$ for glutathione and GSS). (D) Metabolic differences between isogenic renal cell carcinoma RCC4 cell lines identified using 95\% confidence interval bands of regression analysis. (E) Copy number analysis of the $V H L$ gene in cancer cell lines using Cancer Cell Line Encyclopedia data. GSH, glutathione (reduced form); ATP, adenosine triphosphate; GCLC, glutamate-cysteine ligase catalytic subunit; GSS, GSH synthetase; Hsp90, heat shock protein 90; VHL, von Hippel-Lindau tumor suppressor; wt, wild-type; mt, mutant-type; PC, phosphorylcholine; GPC, glycerophosphorylcholine; Asp, aspartic acid; Gln, glutamine.
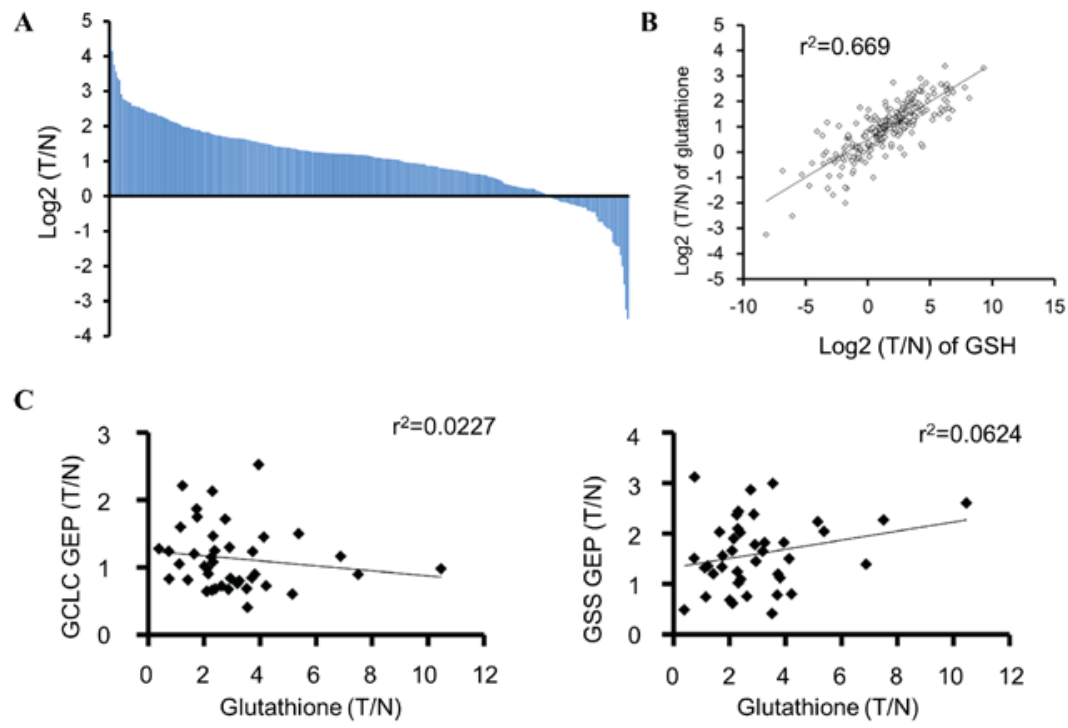

Figure 4. Glutathione levels in tumors of patients with colorectal cancer. (A) Waterfall plots of $\log _{2}(\mathrm{~T} / \mathrm{N})$ values of total glutathione (GSH+GSSG) in tissue samples from patients with colorectal cancer. (B) Correlation between $\log _{2}(\mathrm{~T} / \mathrm{N})$ values of GSH and total glutathione (GSH+GSSG) in tissue samples from patients with colorectal cancer $\left(\mathrm{P}=8.18 \times 10^{-66}\right)$, as evaluated by Pearson correlation analysis. (C) Correlation between total glutathione $(\mathrm{GSH}+\mathrm{GSSG})$ and $\mathrm{GCLC}(\mathrm{P}=0.35)$ or GSS $(\mathrm{P}=0.12)$ mRNA levels $(\mathrm{T} / \mathrm{N})$ in tissue samples from patients with colorectal cancer, as evaluated by Pearson correlation analysis. $\mathrm{T} / \mathrm{N}$, tumor/normal tissues; GSH, glutathione (reduced form); GSSG, glutathione disulphide; GCLC, glutamate-cysteine ligase catalytic subunit; GSS, GSH synthetase; GEP, gene expression. 
may enhance the anticancer effects of GCLC inhibitors. By contrast, HT-29 cells demonstrated more marked combination effects of BSO and iron, although they were less sensitive to the treatment with BSO alone in comparison with PANC-1 cells. There may have been differences in the intracellular iron concentration between PANC-1 and HT-29, which may have caused the differences observed in their sensitivity to the treatment. Additional studies are required to elucidate the mechanism underlying the differences in sensitivity. Notably, in SW48 cells, NAC exerted rescue effects whereas ferrostatin-1 did not, indicating that BSO may induce cell death in this cell line by a ferroptosis-independent mechanism. In conclusion, the present study demonstrated that GCLC inhibition induces ferroptosis in cancer cells, and low glutathione tumor levels may be used as a sensitivity marker for GCLC inhibitors.

\section{Acknowledgements}

The authors would like to thank the following employees of Takeda Pharmaceutical Company Limited: Mr. Shunsuke Ebara for technical assistance, Dr Akito Kadotani and Mr. Shinichi Matsumoto for performing the GCLC enzymatic assay, and Mr. Koji Yamamoto for providing bioinformatics analysis.

\section{Funding}

Takeda Pharmaceutical Company Limited, Japan, supported the present study. The present study was also supported by funds from the AMED-CREST from the Japan Agency for Medical Research and Development, AMED and research funds from the Yamagata prefectural government and the City of Tsuruoka.

\section{Availability of data and materials}

The data sets generated and/or analyzed during the present study are available from the corresponding author upon reasonable request.

\section{Authors' contributions}

SN and TH conceived and designed the experiments. SN, HA, YI, SK, AH and TS performed the experiments. All authors analyzed the data. TS contributed reagents, materials and analysis tools. SN drafted the manuscript. TH reviewed the manuscript. All authors read, revised and approved the final version of the manuscript.

\section{Ethics approval and consent to participate}

All the experiments were conducted according to a study protocol approved by the Institutional Ethics Committee of Kagawa University (Heisei 24-040) upon obtaining informed consent from all subjects.

\section{Consent for publication}

All the experiments were conducted according to a study protocol approved by the Institutional Ethics Committee of Kagawa University (Heisei 24-040) upon obtaining informed consent from all subjects.

\section{Competing interests}

Mr. Satoru Nishizawa, Mr. Hideo Araki, Dr Yoshinori Ishikawa, Mr. Satoshi Kitazawa, Mr. Akito Hata, and Dr Takahito Hara are employees of Takeda Pharmaceutical Company Limited, Japan, which supported the present study.

\section{References}

1. Warburg O: On the origin of cancer cells. Science 123: 309-314, 1956.

2. Vander Heiden MG, Cantley LC and Thompson CB: Understanding the Warburg effect: The metabolic requirements of cell proliferation. Science 324: 1029-1033, 2009.

3. Cairns RA, Harris IS and Mak TW: Regulation of cancer cell metabolism. Nat Rev Cancer 11: 85-95, 2011.

4. Hakimi AA, Reznik E, Lee CH, Creighton CJ, Brannon AR, Luna A, Aksoy BA, Liu EM, Shen R, Lee W, et al: An integrated metabolic atlas of clear cell renal cell carcinoma. Cancer Cell 29: 104-116, 2016

5. Denkert C, Budczies J, Weichert W, Wohlgemuth G, Scholz M, Kind T, Niesporek S, Noske A, Buckendahl A, Dietel $\mathrm{M}$ and Fiehn O: Metabolite profiling of human colon carcinoma-deregulation of TCA cycle and amino acid turnover. Mol Cancer 7: 72, 2008

6. Wang H, Wang L, Zhang H, Deng P, Chen J, Zhou B, Hu J, Zou J, Lu W, Xiang P, et al: ${ }^{1} \mathrm{H}$ NMR-based metabolic profiling of human rectal cancer tissue. Mol Cancer 12: 121, 2013.

7. Li B, Qiu B, Lee DS, Walton ZE, Ochocki JD, Mathew LK, Mancuso A, Gade TP, Keith B, Nissim I and Simon MC: Fructose-1,6-bisphosphatase opposes renal carcinoma progression. Nature 513: 251-255, 2014.

8. Dixon SJ, Lemberg KM, Lamprecht MR, Skouta R, Zaitsev EM, Gleason CE, Patel DN, Bauer AJ, Cantley AM, Yang WS, et al: Ferroptosis: An iron-dependent form of nonapoptotic cell death. Cell 149: 1060-1072, 2012.

9. Yang WS, SriRamaratnam R, Welsch ME, Shimada K, Skouta R, Viswanathan VS, Cheah JH, Clemons PA, Shamji AF, Clish CB, et al: Regulation of ferroptotic cancer cell death by GPX4. Cell 156: 317-331, 2014.

10. Yang WS and Stockwell BR: Ferroptosis: Death by lipid peroxidation. Trends Cell Biol 26: 165-176, 2016.

11. Skouta R, Dixon SJ, Wang J, Dunn DE, Orman M, Shimada K, Rosenberg PA, Lo DC, Weinberg JM, Linkermann A and Stockwell BR: Ferrostatins inhibit oxidative lipid damage and cell death in diverse disease models. J Am Chem Soc 136: 4551-4556, 2014.

12. Xie Y, Hou W, Song X, Yu Y, Huang J, Sun X, Kang R and Tang D: Ferroptosis: Process and function. Cell Death Differ 23: 369-379, 2016.

13. Lu SC: Glutathione synthesis. Biochim Biophys Acta 1830: 3143-3153, 2013.

14. Bailey HH, Ripple G, Tutsch KD, Arzoomanian RZ, Alberti D, Feierabend C, Mahvi D, Schink J, Pomplun M, Mulcahy RT and Wilding G: Phase I study of continuous-infusion L-S,R-buthionine sulfoximine with intravenous melphalan. J Natl Cancer Inst 89: 1789-1796, 1997

15. Sakamoto K, Adachi Y, Komoike Y, Kamada Y, Koyama R, Fukuda Y, Kadotani A, Asami T and Sakamoto JI: Novel DOCK 2-selective inhibitory peptide that suppresses B-cell line migration. Biochem Biophys Res Commun 483: 183-190, 2017.

16. Winterbourn CC and Brennan SO: Characterization of the oxidation products of the reaction between reduced glutathione and hypochlorous acid. Biochem J 326: 87-92, 1997.

17. Winkler BS, DeSantis $\mathrm{N}$ and Solomon F: Multiple NADPH-producing pathways control glutathione (GSH) content in retina. Exp Eye Res 43: 829-847, 1986.

18. Patra KC and Hay N: The pentose phosphate pathway and cancer. Trends Biochem Sci 39: 347-354, 2014.

19. Muñoz-Pinedo C, El Mjiyad N and Ricci JE: Cancer metabolism: Current perspectives and future directions. Cell Death Dis 3: e248, 2012.

20. Singh D, Arora R, Kaur P, Singh B, Mannan R and Arora S: Overexpression of hypoxia-inducible factor and metabolic pathways: Possible targets of cancer. Cell Biosci 7: 62, 2017. 
21. Courtnay R, Ngo DC, Malik N, Ververis K, Tortorella SM and Karagiannis TC: Cancer metabolism and the Warburg effect: The role of HIF-1 and PI3K. Mol Biol Rep 42: 841-851, 2015

22. Haase VH: The VHL tumor suppressor: Master regulator of HIF Curr Pharm Des 15: 3895-3903, 2009.

23. Satoh K, Yachida S, Sugimoto M, Oshima M, Nakagawa T, Akamoto S, Tabata S, Saitoh K, Kato K, Sato S, et al: Global metabolic reprogramming of colorectal cancer occurs at adenoma stage and is induced by MYC. Proc Natl Acad Sci USA 114: E7697-E7706, 2017.

24. Yuan M, Breitkopf SB, Yang X and Asara JM: A positive/negative ion-switching, targeted mass spectrometry-based metabolomics platform for bodily fluids, cells, and fresh and fixed tissue. Nat Protoc 7: 872-881, 2012.

25. Soga T and Heiger DN: Amino acid analysis by capillary electrophoresis electrospray ionization mass spectrometry. Anal Chem 72: 1236-1241, 2000.

26. Soga T, Ohashi Y, Ueno Y, Naraoka H, Tomita M and Nishioka T: Quantitative metabolome analysis using capillary electrophoresis mass spectrometry. J Proteome Res 2: 488-494, 2003.

27. Soga T, Igarashi K, Ito C, Mizobuchi K, Zimmermann HP and Tomita M: Metabolomic profiling of anionic metabolites by capillary electrophoresis mass spectrometry. Anal Chem 81: 6165-6174, 2009.
28. Bailey HH, Mulcahy RT, Tutsch KD, Arzoomanian RZ, Alberti D, Tombes MB, Wilding G, Pomplun M and Spriggs DR: Phase I clinical trial of intravenous L-buthionine sulfoximine and melphalan: An attempt at modulation of glutathione. J Clin Oncol 12: 194-205, 1994.

29. Mehta S, Shelling A, Muthukaruppan A, Lasham A, Blenkiron C, Laking $\mathrm{G}$ and Print C: Predictive and prognostic molecular markers for cancer medicine. Ther Adv Med Oncol 2: 125-148, 2010.

30. Dienstmann R, Rodon J and Tabernero J: Biomarker-driven patient selection for early clinical trials. Curr Opin Oncol 25: 305-312, 2013.

31. Schmidt KT, Chau CH, Price DK and Figg WD: Precision oncology medicine: The clinical relevance of patient-specific biomarkers used to optimize cancer treatment. J Clin Pharmacol 56: 1484-1499, 2016.

32. Terpstra M, Vaughan TJ, Ugurbil K, Lim KO, Schulz SC and Gruetter R: Validation of glutathione quantitation from STEAM spectra against edited 1H NMR spectroscopy at 4T: Application to schizophrenia. MAGMA 18: 276-282, 2005. 